\title{
CONTRIBUTION A L'ÉTUDE DE LA BIOLOGIE
} D'HERIADES TRUNCORUM L.

\author{
(Hymenoptera; Apoidea; Megachilidae)** \\ II - ASPECT ÉCOLOGIQUE \\ Beitrag zur Untersuchung der Biologie \\ von Heriades truncorum $\mathbf{L}$. \\ (Hymenoptera; Apoidea; Megachilidae) \\ II - Ökologischer Aspekt
}

\author{
Maria de Lourdes MACIEL DE ALMEIDA CORREIA* \\ Laboratoire d'Éthologie et d'Écologie des Insectes - I.N.R.A. 86600 Lusignan
}

\section{RÉSUMÉ} que.

Poursuivant l'étude sur la biologie d'Heriades truncorum L., on aborde maintenant l'aspect écologi-

Les femelles apparaissent comme très sélectives dans le choix des nichoirs d'après leur orientation et le choix des tubes de nidification en fonction de leur diamètre. Elles recherchent plutôt les nichoirs orientés vers le sud et l'est, et nidifient de préférence dans des tubes de $3 \mathrm{~mm}$ de diamètre.

Heriades truncorum L. a un comportement typiquement oligolectique vis-à-vis de la flore (Elle est strictement inféodée aux Composées.) Près de $57 \%$ des cellules contiennent des provisions uniflorales $(56,7 \%)$. Les provisions à $2,3,4$ ou 5 pollens représentent respectivement $23,5 \%, 15 \%, 4,3 \%$ et $0,5 \%$ du total des cellules. Les Composées à fleurs jaunes, dont les pollens sont petits $(23$ à $32 \mu \mathrm{m})$ et de couleur jaune, sont nettement préférées, particulièrement Senecio, Inula, Crepis et Picris. Les deux premières ont des pollens du type "échinulé ", les deux autres du type "fenestré ".

$\left({ }^{*}\right)$ Boursière de la Fondation Calouste Gulbenkian.

$\left({ }^{* *}\right)$ Ce travail constitue une partie d'une thèse de Doctorat d'État ès Sciences soutenue le 11 janvier 1979 devant l'Université de Pierre et Marie Curie - Paris-VI. 


\author{
SUMMARY \\ STUDY OF THE BIOLOGY OF HERIADES TRUNCORUM L. \\ (HYM., APOIDEA, MEGACHILIDAE.) \\ II. - Ecological point of view
}

This study reports the ecological aspects of our studies on the biology of Heriades truncorum L.

The females appeared to be rather selective in their choice of the orientation and diameter of the nest cavities. They preferred nests facing south and east, and tubes $3 \mathrm{~mm}$ in diameter.

Heriades truncorum L. showed a typical oligolectic behaviour towards the family Compositae. Almost $57 \%$ of the cells contained a unifloral supply $(56,7 \%)$. Other cells were provisioned with $2,3,4$ and 5 types of pollens respectively in $23,5 \%, 15 \%, 4,3 \%$ and $0,5 \%$ of the cells. Small (23 to $32 \mu \mathrm{m})$ and yellow pollens were clearly preferred, namely from Senecio, Inula, Crepis and Picris; the first two genera represent pollens of the "echinule "type, and the others have "fenestre " type.

\title{
INTRODUCTION
}

Dans un article précédent (Maciel Correia, sous presse) le cycle biologique de l'espèce Heriades truncorum L. a été observé. Son caractère monovoltin est mis en évidence et les différents stades larvaires sont étudiés avec précision.

La présente note fait état de l'influence du milieu sur le choix du site de nidification. On a observé l'influence de l'orientation des nichoirs et du diamètre des tubes de nidification sur le comportement des femelles.

Par ailleurs, la nidification étant plus abondante dans certains lieux par rapport à d'autres, nous analysons le choix alimentaire en fonction du milieu. Il s'est avéré indispensable de connaître les associations écologiques existant entre les plantes fréquentes dans la région d'observation et les insectes qui les visitent.

Dans une première phase, on procède à un inventaire de la flore environnante des sites de nidification dans un rayon de 200 à $300 \mathrm{~m}$; cela permet de mettre en évidence les espèces caractéristiques de chaque milieu. Dans une deuxième phase et grâce à l'analyse pollinique des provisions de pollen des cellules des nids prélevés aux divers endroits de piégeage on décèle les espèces sur lesquelles les femelles récoltent le pollen. Cette recherche fait suite à notre travail concernant les sources des matériaux de construction des nids (Maciel Correia, 1977).

\section{MATÉRIEL ET MÉTHODES}

Nos recherches ont été poursuivies sur sept emplacements dans la région de Lusignan et un emplacement dans la région de Barbezieux pendant quatre années consécutives (1974-1977). Quelques observations complémentaires concernant les années 1972 et 1973 nous ont été fournies par le Laboratoire de Zoologie de Lusignan, ce qui nous a permis d'étudier l'évolution des populations des Heriades sur une durée de six ans. 


\section{- PRÉSENTATION DES SITES NATURELS PROSPECTÉS}

Le choix des lieux prospectés a été fait sur des cartes de la région de Lusignan au 1/50000 (MACleL CoRreia, 1976 a) représentant différents types d'habitats. Un seul endroit, Barbezieux, se situe à $150 \mathrm{~km}$ au sud de Lusignan.

- La Girauderie (Jazeneuil), situé sur un haut plateau (144 $\mathrm{m}$ d'altitude) près d'un champ cultivé, a un sol sain. Il se trouve en bordure d'un reliquat forestier formé de taillis sous futaie. C'est un groupement de chênaie-charmaie, à base de charme (Carpinus betulus L.), de chêne (Quercus pedunculata Ehrh.), châtaignier (Castanea sativa Miller), érable (A cer campestre L.), orme (Ulmus). Le sous-bois est réduit à un fourré de prunelliers (Prunus), cerisiers sauvages (Prunus cerasus L.), aubépines (Crataegus), églantiers (Rosa canina L.). Les dimensions des parcelles cultivées varient de 2 à 3 ha. Nos nichoirs-pièges sont accrochés aux murs d'une cabane en planches de pin de dimensions $4 \times 2 \times 2 \mathrm{~m}$ bâtie dans un angle d'un champ de 2 ha entouré de haies constituées de noisetiers (Corylus avellana L.), noyers (Juglans regia L.), frênes (Fraxinus), sureaux (Sambucus nigra L.), épines noires (Prunus spinosa L.), clématites (Clematis vitalba L.), ormeaux taillés (Ulmus campestris L.). On trouve aussi des futaies résineuses installées artificiellement et constituées de Pseudotsuga douglasii Carr. (pin importé d'Amérique du Nord).

- Mongadon situé à $108 \mathrm{~m}$ d'altitude est un fond de la vallée légèrement encaissée. Les sols de la dépression sont souvent gorgés d'eau en hiver et au printemps. Le groupement est l'aulnaie : on y rencontre l'aulne (Alnus glutinosa L.), le saule (Salix triandra L.), les plantes aquatiques (Iris pseudacorus L.) et les joncs (Juncus $\mathrm{sp}$.). Plus haut dans les endroits plus secs, on trouve des clématites (Clematis vitalba L.). Les nichoirs sont accrochés à deux cabanes en bois de $5 \times 3 \times 3 \mathrm{~m}$ et de $2 \times 2 \times 3 \mathrm{~m}$ et à des arbres (peupliers). Ces emplacements sont dans une cressonière exploitée sur le fond de la vallée, d'environ $300 \mathrm{~m}$ de largeur. En bordure et à mi-pente, une forêt de chênes (Quercus pedunculata Ehrh.). On trouve aussi l'aubépine (Crataegus sp.), le frêne (Fraximus sp.), le pommier (Malus sp.), l'épine noire (Prunus spinosa L.), des ronces (Rubus sp.). A peu de distance se trouvent des peupliers (Populus alba L.). Sur le coteau la chênaie domine.

- Servolet (133 m d'altitude) est situé dans une ancienne carrière, l'emplacement a une physionomie complètement différente de celle des autres. Cette zone abritée est caractérisée par des terrains calcaires, très secs et rocailleux. Le peuplement est formé de Robinia pseudo-acacia L., le sous-bois de ronces (Rubus sp.). Le plateau est à $15 \mathrm{~m}$ au-dessus du fond de la carrière. L'excavation est de forme ovale, de $100 \mathrm{~m}$ de large et de $30 \mathrm{~m}$ de profondeur. Les nichoirs sont accrochés dans des anfractuosités de la paroi verticale. Les environs de la carrière sont des bois, des prés et des cultures variées exploitées en parcelles de 2 à 4 ha.

- Le Chêne à $145 \mathrm{~m}$ d'altitude est un domaine de 50 ha d'un seul tenant dépourvu d'arbres (quelques jeunes noyers et trois vieux chênes morts) sur lequel sont cultivées diverses plantes fourragères, du maïs, du colza, du blé. Les environs sont soit des bois, soit des prés ou des cultures de 1 à 3 ha entourés de haies.

Les nichoirs sont accrochés aux murs de la ferme située au milieu du domaine et à un séchoir à maïs en bois. Les murs de la ferme sont recouverts d'un crépis mais certains corps de bâtiment sont en pierre apparente.

On a également fixé quelques nichoirs au tronc de châtaigniers morts à $50 \mathrm{~m}$ de la ferme. En bordure, la présence d'une haie formée de ronces, d'aubépines, d'épines noires, de lierre et d'érables. A environ $100 \mathrm{~m}$ se trouve une bordure de conifères surtout de Thuva.

- La Pétinière et les Verrines sont des domaines semblables au Chêne. La surface de la Pétinière est inférieure à celle du Chêne ( 10 ha d'un seul tenant). Le type de végétation est identique au Chêne; l'altitude varie de $141 \mathrm{~m}$ à la Pétinière, à $152 \mathrm{~m}$ aux Verrines.

- Le Grand-Breuil est un hameau de maisons en pierres apparentes liées par un mélange chaux et terre. Les environs sont des prés et des champs de 2 à 3 ha entourés par des haies. Le hameau est situé sur le plateau à $134 \mathrm{~m}$ d'altitude.

- Barbezieux situé dans la Charente est une plaine vallonnée et cultivée. Les nichoirs sont accrochés aux montants d'un séchoir à maïs et aux murs d'une ferme abandonnée située au centre d'un domaine de 50 ha dépourvu de haies. 


\section{TECHNIQUES D'ÉTUDE}

\section{Recensement des nids}

L'accessibilité des nids naturels étant très aléatoire, nous avons employé la méthode de piégeage décrite dans une note précédente (MACIEL CorReIA, sous presse). Afin de suivre l'activité des insectes, nous avons effectué des contrôles très réguliers et le prélèvement journalier des nids.

Nos observations ont été effectuées dans les conditions climatiques de la région de Lusignan. Les données météorologiques ont été recueillies sur place dans le poste local de météorologie (I.N.R.A., Les Verrines; altitude $=150 \mathrm{~m}$, latitude 51,60 grades; longitude $=-0,15$ grades).

\section{Abondance relative des espèces végétales et choix des fleurs butinées}

- Inventaire floristique.

Il est effectué dans les lieux de piégeage dans un rayon de $300 \mathrm{~m}$ par identification précise des espèces végétales rencontrées, en tenant compte uniquement des espèces susceptibles d'avoir un intérêt pour le travail entrepris. Un grand nombre de familles (26) et d'espèces (81) sont représentées au total dans nos lieux de piégeage.

- Analyse pollinique des provisions des nids.

Méthode de montage des préparations microscopiques.

Trois échantillons de pollen sont généralement prélevés avec une aiguille lancéolée à différents endroits de chaque cellule. Le premier prélèvement est proche de la cloison, le second est au centre de la provision de pollen et le troisième est proche de l'œuf. Les trois échantillons sont traités séparément et servent à la confection de préparations microscopiques. Ce prélèvement doit être effectué le plus tôt possible, dès que les nids sont achevés, afin d'éviter une certaine modification des pollens due aux sécrétions salivaires des femelles qui "digèrent " le cytoplasme et altèrent la forme générale du grain (TASEI, 1973).

Le traitement de ces échantillons s'effectue selon une méthode proche de celle qui est recommandée par Louveaux et MaURIzıo (1963) en mélissopalynologie. Le pollen est dilué et lavé à l'eau distillée (1 à 2 gouttes). Ensuite une quantité minime (1 goutte) est étalée sur une lame porte-objet posée sur une plaque chauffante à $50^{\circ} \mathrm{C}$. Après évaporation de l'eau, on dégraisse le pollen à l'aide d'éther que l'on fait couler sur la lame à plusieurs reprises. Finalement, le pollen convenablement dégraissé est inclus dans la gélatine glycérinée légèrement colorée à la fuchsine et liquéfiée à la chaleur. L'examen de ces préparations s'effectue par comparaison avec les lames de référence existant dans le laboratoire. L'identification des pollens est facilitée par la connaissance de la flore locale (espèces présentes et dates de floraison dans les lieux de piégeage) et par la connaissance de la date d'approvisionnement des nids.

On commence par un inventaire des espèces contenues dans la préparation. L'évaluation quantitative est ainsi faite : pour chaque préparation on attribue à chaque pollen présent une note de "volume occupé " tenant compte de la fréquence et du diamètre du grain. La note varie de 1 à 10 . Le total des notes attribuées aux pollens d'une même préparation est toujours égal à 10 . Cette estimation volumétrique relativement rapide s'est révélée d'une bonne précision puisque l'opérateur a toujours donné les mêmes proportions pour les trois prélèvements d'une même cellule, alors qu'il était dans l'ignorance de leur origine.

Nous avons ainsi analysé le contenu de 208 cellules. Le regroupement des notes en fonction de l'origine des préparations a permis de calculer les proportions des volumes de pollen de chaque espèce qui ont servi aux approvisionnements, selon le lieu, l'année, le mois.

\section{RÉSULTATS}

Nous avons obtenu pour l'ensemble des emplacements et pour six années d'observations 208 nids, soit 585 cellules. Chaque nid est formé d'une ou plusieurs cellules disposées linéairement (voir l'architecture du nid dans une note précédente). Nos résultats sont ainsi exprimés en nombre de cellules contenant des œufs. 


\section{Choix des nichoirs-pièges d'après leur orientation}

Nous avons testé huit orientations pendant six ans dans sept lieux de piégeage sur les huit déjà mentionnés. Afin d'uniformiser les conditions de l'observation, nous avons proposé le même nombre de nichoirs à chaque orientation en chaque lieu. On a ainsi disposé chaque année un total de 10 nichoirs (soit 150 tubes de nidification) à chacune des orientations. Quatre-vingts nichoirs ont servi à ces observations. Nous avons ainsi mis en évidence qu'Heriades truncorum L. nidifie de préférence dans les nichoirs dont les tubes sont orientés vers le sud et l'est (fig. 1). Les nichoirs orientés au sud-est et

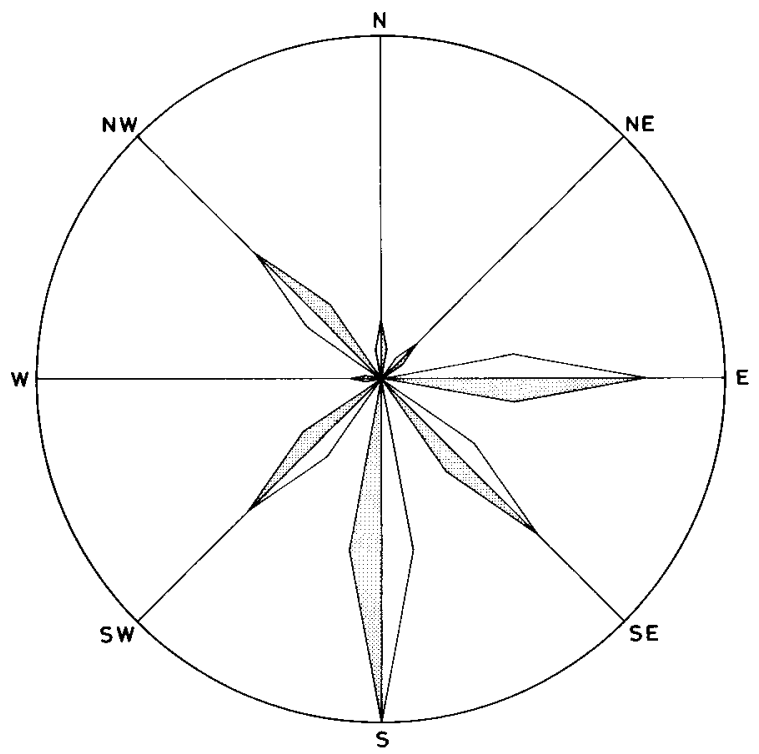

FIG. 1. - Choix des nichoirs-pièges d'après leur orientation.

La longueur de chaque branche est proportionnelle

au pourcentage de nids construits dans les nichoirs orientés vers la direction indiquée.

Авв. 1. - Wahl der gebotenen Köder-Nistplätze nach ihrer Orientierung.

Die Länge jedes Armes ist proportional dem Prozentsatz

der in den nach bestimmten Richtungen orientierten Nistplätzen gebauten Nester.

nord-ouest sont également assez bien acceptés. Ceux qui sont orientés au nord, à l'ouest et au nord-est sont très rarement utilisés par les femelles. Ce comportement peut s'expliquer par le fait que les nichoirs ouest et surtout nord et nord-est sont frappés moins longtemps que les autres par la lumière directe du soleil. Les nichoirs orientés vers le nord-ouest font exception. 


\section{Choix des tubes en fonction de leur diamètre}

Les femelles manifestent une nette préférence pour les tubes d'un diamètre de $3 \mathrm{~mm}$ (tableau 1 et Maciel Correia, 1976). En présence de tubes variant de 2,5 mm à

TABL. 1. - Fréquence de distribution du nombre de cellules par nid en fonction du diamètre. Années 1972 à 1977.

TAB. 1. - Häufigkeit der Verteilung der Zellzahl pro Nest in Abhängigkeit von ihrem Durchmesser (1972-1977).

\begin{tabular}{|c|c|c|c|c|}
\hline \multirow{2}{*}{$\begin{array}{l}\text { Cellules par nid } \\
\text { Zellen je Nest }\end{array}$} & \multicolumn{3}{|c|}{$\begin{array}{l}\text { Nombre de nids } \\
1972-1977 \\
\text { Anzahl der Nester }\end{array}$} & \multirow[t]{2}{*}{ Total } \\
\hline & $3 \mathrm{~mm}$ & $4 \mathrm{~mm}$ & $5 \mathrm{~mm}$ & \\
\hline 1 & 21 & 10 & 1 & 32 \\
\hline 2 & 43 & 5 & 2 & 50 \\
\hline 3 & 41 & 13 & 1 & 55 \\
\hline 4 & 26 & 15 & 2 & 43 \\
\hline 5 & 2 & 5 & 2 & 9 \\
\hline 6 & - & 3 & - & 3 \\
\hline 7 & - & 1 & - & 1 \\
\hline 8 & - & - & 1 & 1 \\
\hline Total & 133 & 52 & 9 & 194 \\
\hline
\end{tabular}

$6 \mathrm{~mm}$ de diamètre qui ont été présentés aux insectes, la nidification préférentielle est obtenue dans ceux de $3 \mathrm{~mm}$ (fig. 2). A noter qu'il s'agit d'une moyenne globale obtenue pour les 7 localités. Au cours de sa vie, une femelle donnée nidifie généralement dans des tubes qui ont tous le même diamètre. Elle inspecte en moyenne 5 trous avant d'en choisir un, et la durée de telles visites varie de 15 à 50 s. Rares sont les visites de 3 à $4 \mathrm{mn}$.

\section{Variations annuelles des populations}

Après une première analyse des nidifications obtenues, on constate que les populations d'Heriades évoluent de façon différente selon les années : les années 1973 et 1977 sont particulièrement propices à la nidification (fig. 3). Les années 1972 et 1974 présentent à peu près le même niveau de nidifications et l'année 1976 s'est révélée très mauvaise.

En ce qui concerne la variation annuelle selon les différentes stations de piégeage, l'observation de la figure 4 permet de conclure que : La Pétinière, Le Chêne et Jazeneuil se trouvent dans le même groupe, avec une nidification plus importante que les autres. Pour La Pétinière et Le Chêne les nidifications sont constantes d'une année sur l'autre. Pendant toute la période des observations, nous avons obtenu des cellules dans ces 


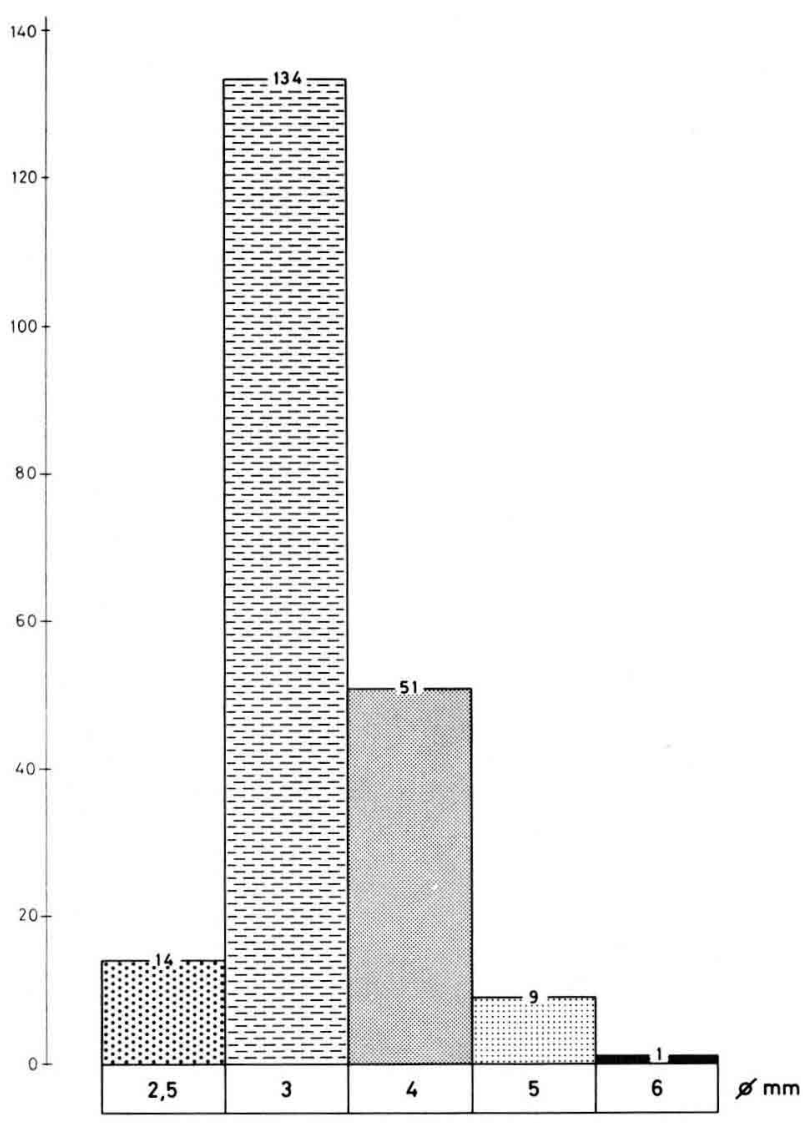

FIG. 2. - Choix des tubes en fonction de leur diamètre.

Les résultats portent sur 209 nids récoltés dans 80 nichoirs chaque année. Verticalement : nombre de nids construits.

ABB. 2. - Wahl der Röhrchen in Abhängigkeit von ihrem Durchmesser. Die Ergebnisse beziehen sich auf 209 Nester, die jährlich in 80 Nistplätzen gesammelt wurden. Vertikal : Anzahl der gebauten Nester.

deux endroits de piégeage (sauf en 1972 au Chêne). Au Chêne, il semble que le nombre de cellules tende à s'accroître. Jazeneuil est l'endroit où l'activité est la plus importante, mais assez irrégulière tout comme à La Pétinière. A Jazeneuil, les années à forte population alternent avec les années à faible population. A Barbezieux, on note une activité nulle pendant deux années consécutives puis une augmentation considérable pendant la troisième, à Mongadon, une nette tendance à la décroissance de l'activité d'année en année. Aux Verrines, Grand-Breuil et Servolet, la nidification est sporadique.

\section{Interprétation statistique}

Nous avons entrepris une analyse statistique du comportement d'Heriades truncorum $\mathrm{L}$. 


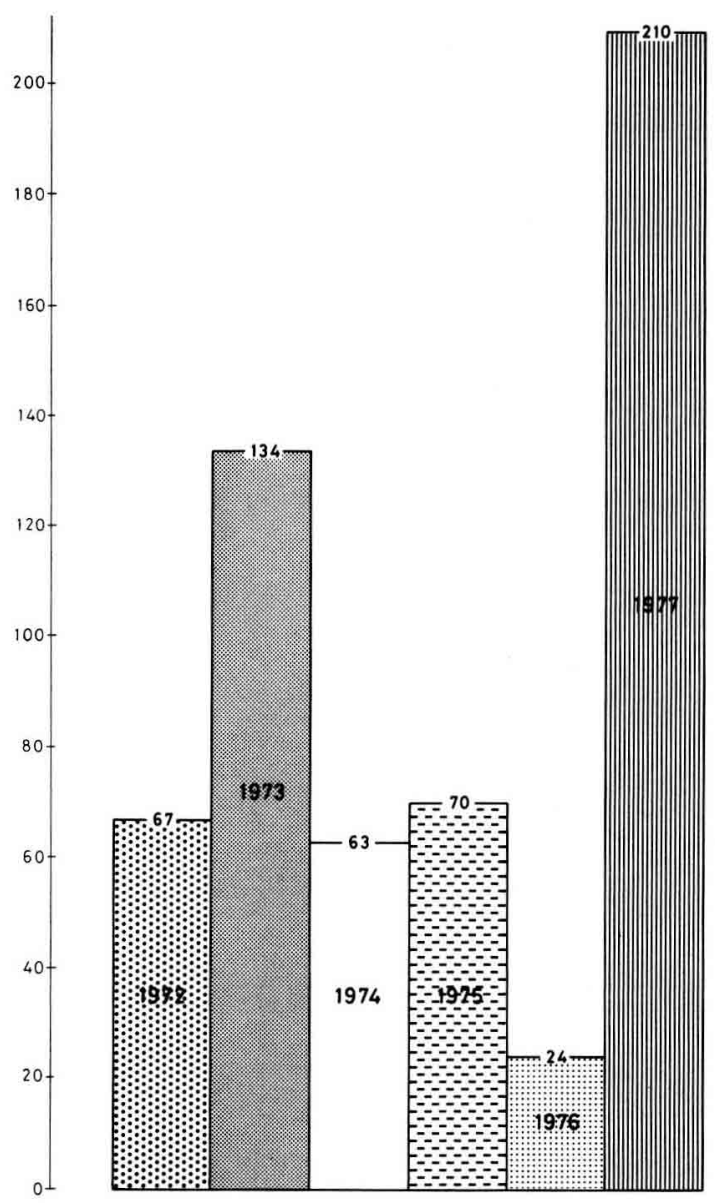

FIG. 3. - Effectifs des cellules récoltées au cours de six années et dans huit localités. Dans chaque localité sont disposés 113 pièges contenant chacun 15 tubes de nidification. Verticalement : nombre de cellules construites.

ABв. 3. - Gesamtzahl der gesammelten Zellen, die im Verlaufe von sechs Jahren an acht Fundorten gesammelt wurden.

An jeder Stelle wurden 113 Nistplätze mit je 15 Niströhrchen aufgestellt. Vertikal : Anzahl der gebauten Zellen.

L'étude porte sur six années et les comptages des nids construits et des cellules approvisionnées ont été effectués dans les huit endroits de piégeage : tableaux 2 et 3 .

L'objet de cette étude est de faire ressortir les effets simples et les interactions au cours des années et des lieux d'observation. Pour tester les effets (années et lieux), nous utilisons l'analyse de l'information appliquée à la loi multinomiale (ARBONNIER, 1967). Pour comparer la nidification et l'approvisionnement en cellules quant à la répartition de la population au cours des années, nous avons utilisé le test de l'homogénéité ou le test de comparaison entre échantillons (ARBONNIER, 1967). 

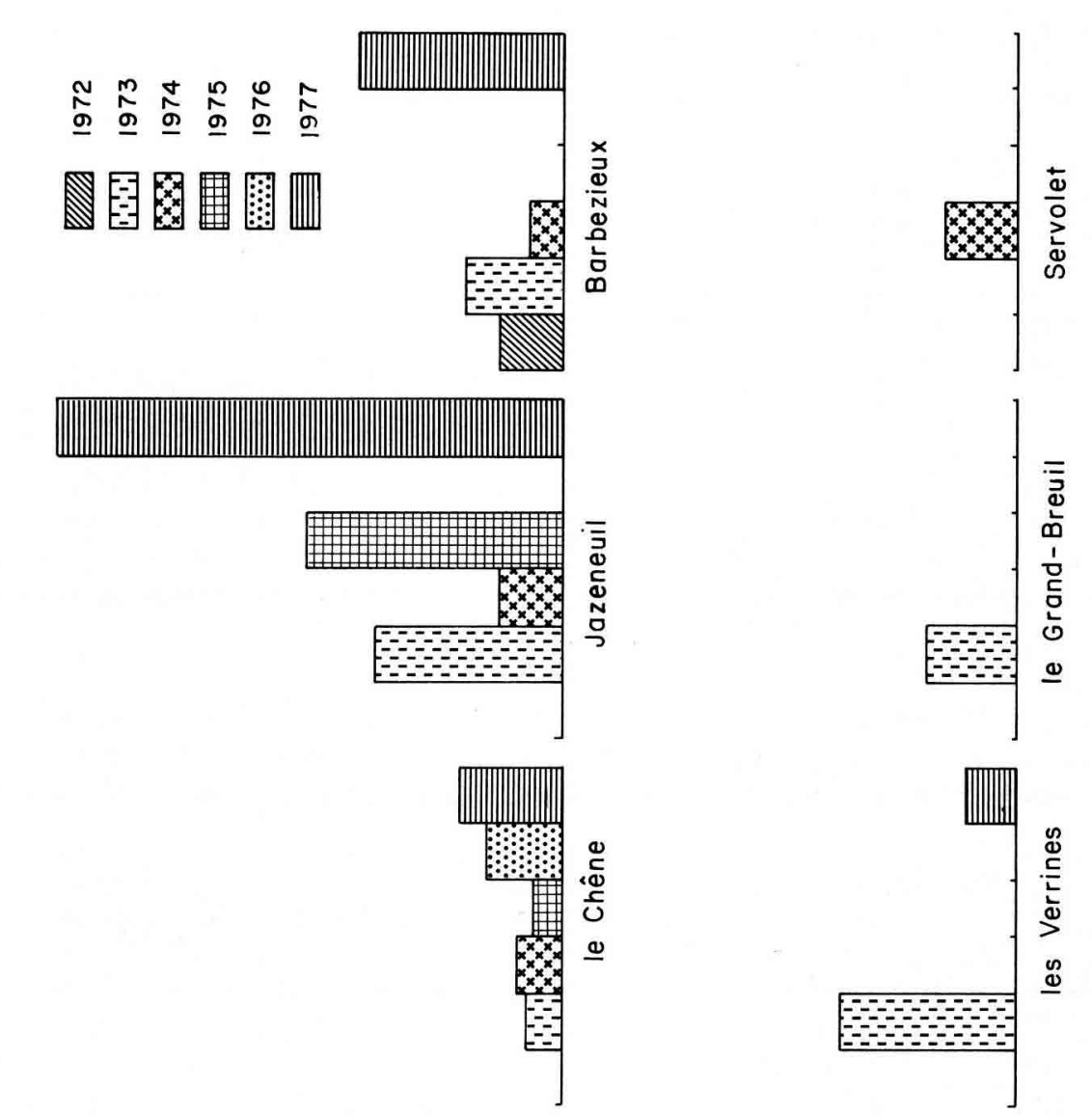

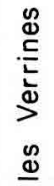
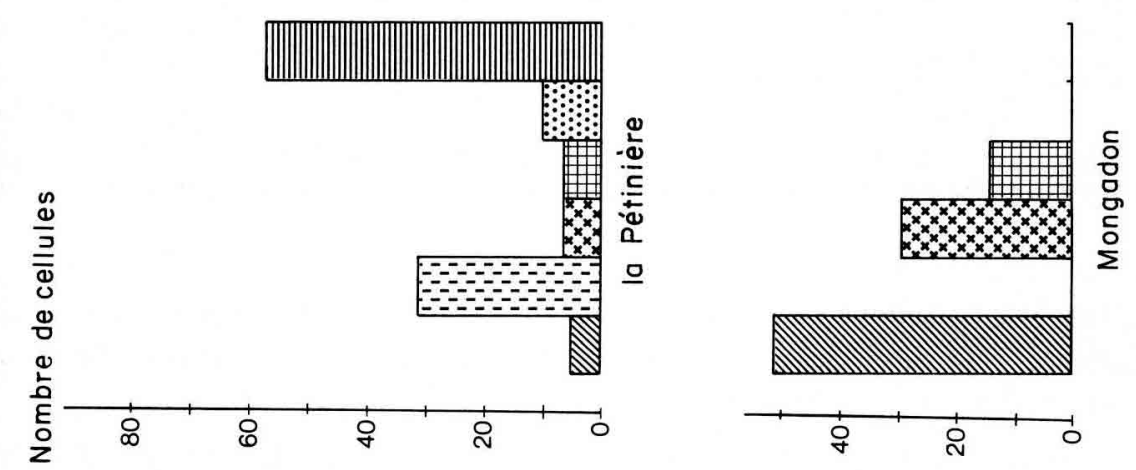

Fig. 4. - Nombre de cellules construites selon les années dans différentes stations.

Les pièges étant disposés en permanence dans toutes les localités on constate que la récolte des cellules a été nulle au cours de certaines années.

Verticalement : nombre de cellules.

Aвв. 4. - Anzahl der gebauten Zellen pro Jahr an den verschiedenen Stationen. Obwohl Köder-Nistplätze an allen Orten ständig aufgestellt waren, wurde festgestellt, dass in manchen Jahren keine Zellen gesammelt werden konnten. Vertikal : Anzahl der Zellen. 
TABL. 2. - Distribution annuelle des populations (nombre de nids) $d$ 'Heriades truncorum $L$. TAB. 2. - Jährliche Verteilung der Populationen (Anzahl der Nester) von Heriades truncorum $L$.

\begin{tabular}{c|c|c|c|c|c|c|c|c|c}
\hline $\begin{array}{r}\text { Lieux } \\
\text { Ort }\end{array}$ & $\begin{array}{c}\text { Barbe- } \\
\text { zieux }\end{array}$ & Chêne & $\begin{array}{c}\text { Jaze- } \\
\text { neuil }\end{array}$ & $\begin{array}{c}\text { Grand- } \\
\text { Breuil }\end{array}$ & $\begin{array}{c}\text { Verri- } \\
\text { nes }\end{array}$ & $\begin{array}{c}\text { Péti- } \\
\text { nière }\end{array}$ & $\begin{array}{c}\text { Servo- } \\
\text { let }\end{array}$ & $\begin{array}{c}\text { Mon- } \\
\text { gadon }\end{array}$ & Total \\
\hline 1972 & 2 & - & - & - & - & 3 & - & 18 & 23 \\
1973 & 4 & 2 & 13 & 6 & 8 & 8 & - & 1 & 42 \\
1974 & 2 & 5 & 6 & - & 1 & 3 & 6 & 10 & 33 \\
1975 & - & 2 & 18 & - & - & 2 & - & 5 & 27 \\
1976 & - & 5 & - & - & - & 5 & - & - & 10 \\
1977 & 9 & 7 & 32 & - & 4 & 21 & - & - & 73 \\
Total & 17 & 21 & 69 & 6 & 13 & 42 & 6 & 34 & 208 \\
\hline
\end{tabular}

TABL. 3. - Distribution annuelle des populations (nombre de cellules) $d$ 'Heriades truncorum $L$.

TAB. 3. - Jährliche Verteilung der Populationen (Anzahl der Zellen) von Heriades truncorum $L$.

\begin{tabular}{|c|c|c|c|c|c|c|c|c|c|}
\hline $\begin{array}{l}\text { Années } \\
\text { Jahr }\end{array}$ & $\begin{array}{l}\text { Barbe- } \\
\text { zieux }\end{array}$ & Chêne & $\begin{array}{l}\text { Jaze- } \\
\text { neuil }\end{array}$ & $\begin{array}{l}\text { Grand- } \\
\text { Breuil }\end{array}$ & $\begin{array}{l}\text { Verri- } \\
\text { nes }\end{array}$ & $\begin{array}{l}\text { Péti- } \\
\text { nière }\end{array}$ & $\begin{array}{l}\text { Servo- } \\
\text { let }\end{array}$ & $\begin{array}{l}\text { Mon- } \\
\text { gadon }\end{array}$ & Total \\
\hline 1972 & 11 & - & - & - & - & 5 & - & 51 & 67 \\
\hline 1973 & 17 & 6 & 33 & 16 & 31 & 31 & - & 3 & 137 \\
\hline 1974 & 6 & 8 & 11 & - & 4 & 6 & 13 & 29 & 77 \\
\hline 1975 & - & 5 & 45 & - & - & 6 & - & 14 & 70 \\
\hline 1976 & - & 14 & - & - & - & 10 & - & - & 24 \\
\hline 1977 & 36 & 19 & 89 & - & 9 & 57 & - & - & 210 \\
\hline Total & 70 & 52 & 178 & 16 & 44 & 115 & 13 & 97 & 585 \\
\hline
\end{tabular}

Ainsi, nous pouvons conclure que d'une année à l'autre, le niveau des populations en cours de nidification est significativement différent et que le nombre de cellules approvisionnées est très variable.

Sur l'ensemble des six années, il y a des différences très significatives entre lieux, que ce soit pour la nidification ou pour le nombre de cellules approvisionnées. On s'aperçoit que la population est très hétérogène, comme si les facteurs de la dynamique de la population variaient différemment au cours du temps, d'un lieu à l'autre. L'approvisionnement de cellules varie au cours des années et des lieux d'expérimentation; on peut donc conclure a priori que d'autres facteurs interviennent sur le nombre de cellules construites par l'insecte. 
4. Variations mensuelles des populations

D'après les histogrammes des variations numériques globales au cours de l'année (fig. 5) établis en groupant toutes les données, nous constatons un début d'activité en

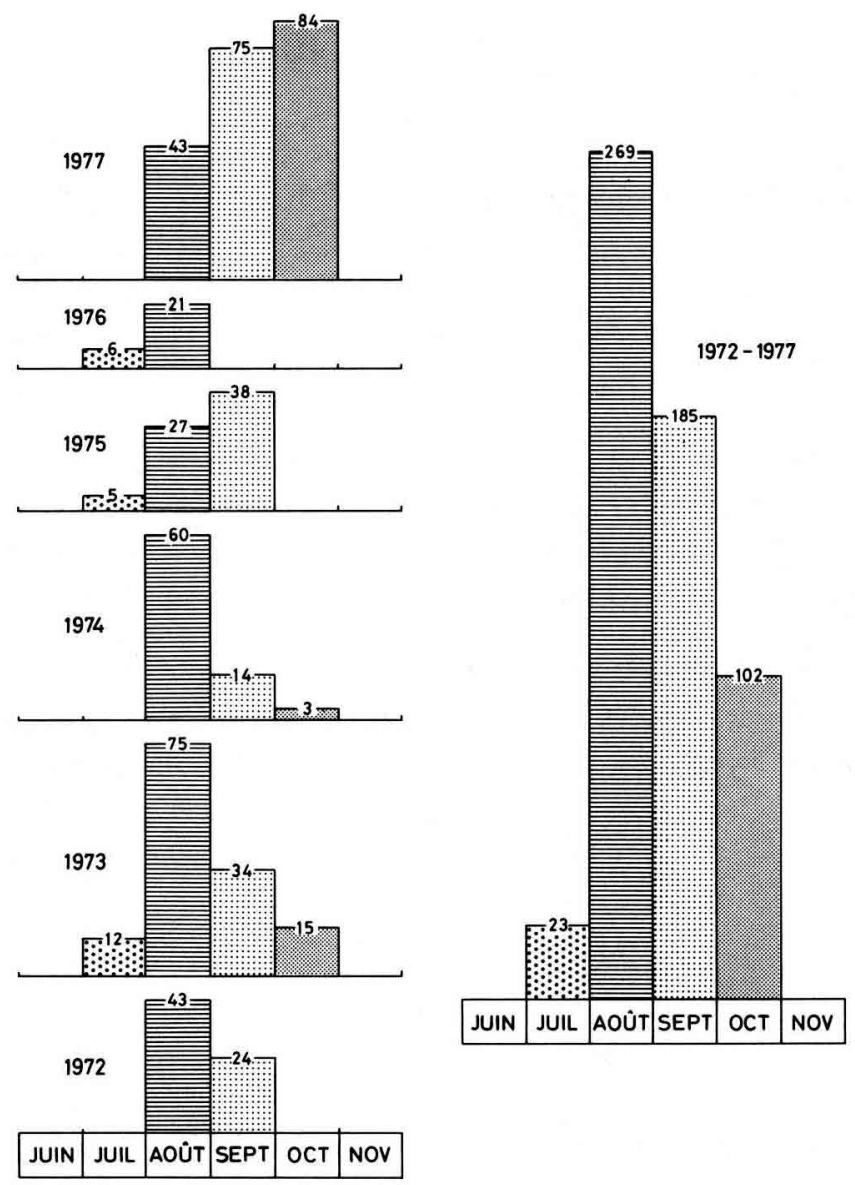

FIG. 5. - Variations mensuelles des nidifications $d$ 'Heriades truncorum $L$.

Авв. 5. - Monatliche Schwankungen der Nestgründung von Heriades truncorum $L$.

juillet avec un maximum en août. Le nombre de nidifications diminue en septembre pour s'annuler au plus tard à la mi-octobre. Ce résultat est bien évident sur la figure 6 .

- Variations mensuelles en fonction des années.

L'observation des histogrammes établis pour chaque année confirme le début des nidifications en juillet et un maximum de nids généralement en août (fig. 5). Toutefois pour l'année 1975, ce maximum est en septembre et en 1977 il est en octobre. Pendant les deux années 1973 et 1977 qui se sont révélées comme de bonnes années, l'échelon- 


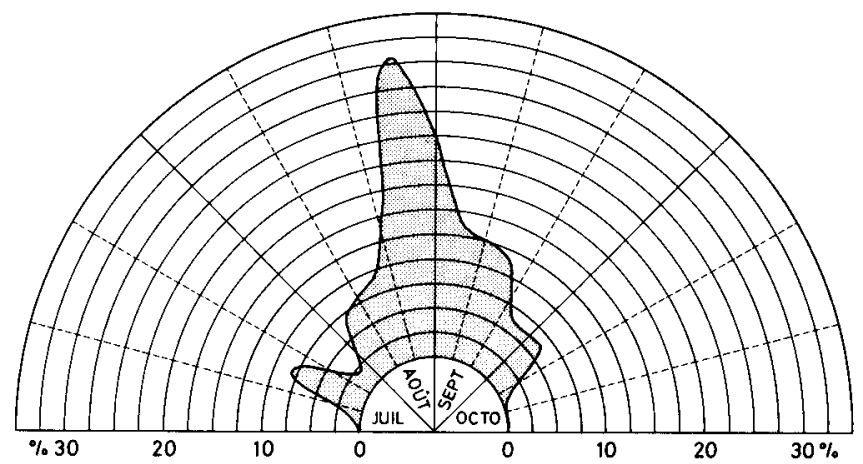

FIG. 6. - Variation par décade de la nidification d'Heriades truncorum $L$. AвB. 6. - Schwankung der Nestgründung von Heriades truncorum L. pro Dekade.

nement des nidifications varie un peu : en 1973 l'activité débute en juillet, augmente rapidement pour atteindre son maximum en août et diminuer progressivement en septembre et octobre. Par contre, en 1977, elle commence en août, augmente en septembre, atteint son maximum début octobre et s'arrête complètement le mois suivant.

- Variations mensuelles suivant les endroits de piégeage.

Sur l'ensemble des années (fig. 7), nous pouvons conclure que Jazeneuil est l'endroit où le nombre de cellules construites a été le plus élevé. Ensuite viennent La Péti-

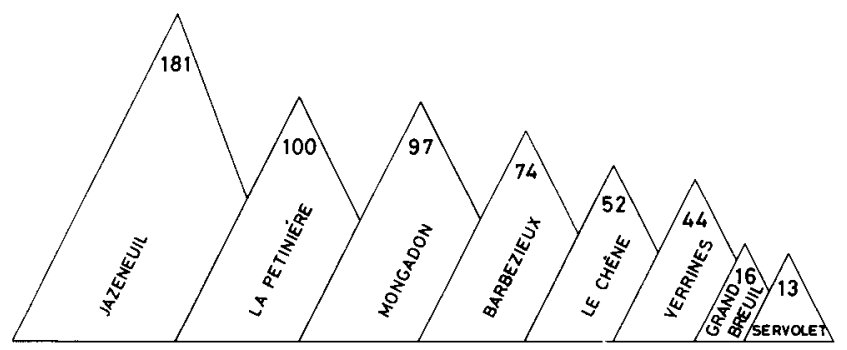

Fig. 7. - Provenance des cellules récoltées au cours des six années d'observation. Aвв. 7. - Herkunft der gewonnenen Zellen im Verlauf der 6 Beobachtungsjahre.

nière et Mongadon presque au même niveau, suivis de Barbezieux, Le Chêne, Les Verrines. Le Grand-Breuil et Servolet se révèlent comme les moins riches.

En analysant plus en détail la répartition des cellules construites selon les lieux (fig. 8), Jazeneuil se révèle toujours l'emplacement le plus favorable, en nombre et en étalement de la période d'activité (de juillet à octobre). Mongadon présente un pic en août et à La Pétinière l'activité s'échelonne de juillet à octobre, mais présente deux pics, en août et octobre. 

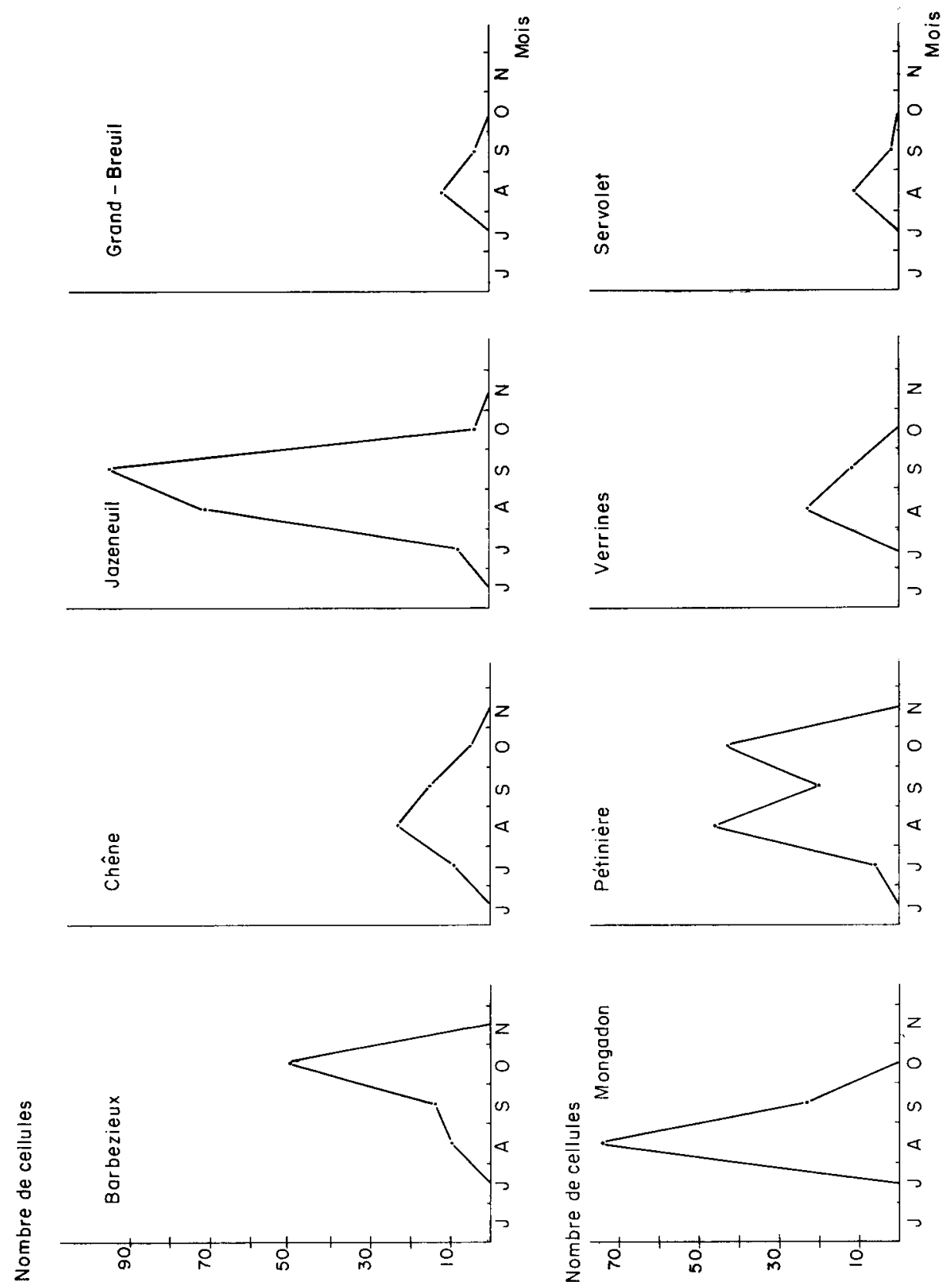

FIG. 8. - Variations mensuelles de l'activité de nidification suivant les stations de piégeage au cours des années 1972 à 1977.

Verticalement : nombre de cellules.

ABв. 8. - Zentrale Schwankungen der Nistaktivität je Köder-Station im Verlaufe der Jahre 1972-1977.

Vertikal : Anzahl der Zellen. 
Nous avons voulu voir s'il existait une corrélation entre cette intensité de nidification et les températures moyennes maxima, minima et la pluviométrie par décade. Toutefois, cette étude ne nous permet pas de déceler l'influence directe de ces facteurs sur la nidification. Il faut remarquer que les corrélations étudiées se rapportent à des données météorologiques concernant le climat moyen de la région. Il est possible que des facteurs du microclimat interviennent également.

Nous pensons que certains facteurs du milieu ont pu jouer un rôle aussi important, sinon plus, que les facteurs climatiques, par exemple l'abondance des plantes nourricières et l'accessibilité du pollen et du nectar, ou celles des plantes fournissant le matériau de construction.

\section{Pollens récoltés par les femelles}

Par l'observation de tous les échantillons prélevés aux huit endroits de piégeage, nous avons retrouvé les diverses espèces végétales butinées par l'insecte telles que : Senecio vulgaris L., Lagoseris nemausensis Cass., Calendula sp., Carduus sp., Sonchus asper All., Cirsium lanceolatum Scop., Crepis virens L., Senecio jacobaea L., Centaurea pratensis Thoill., Cichorium intybus L., Cirsium arvense Scop., Inula dysenterica L., Picris hieracoïdes L., Eupatorium cannabinum L., Lappa sp. (fig. 9).
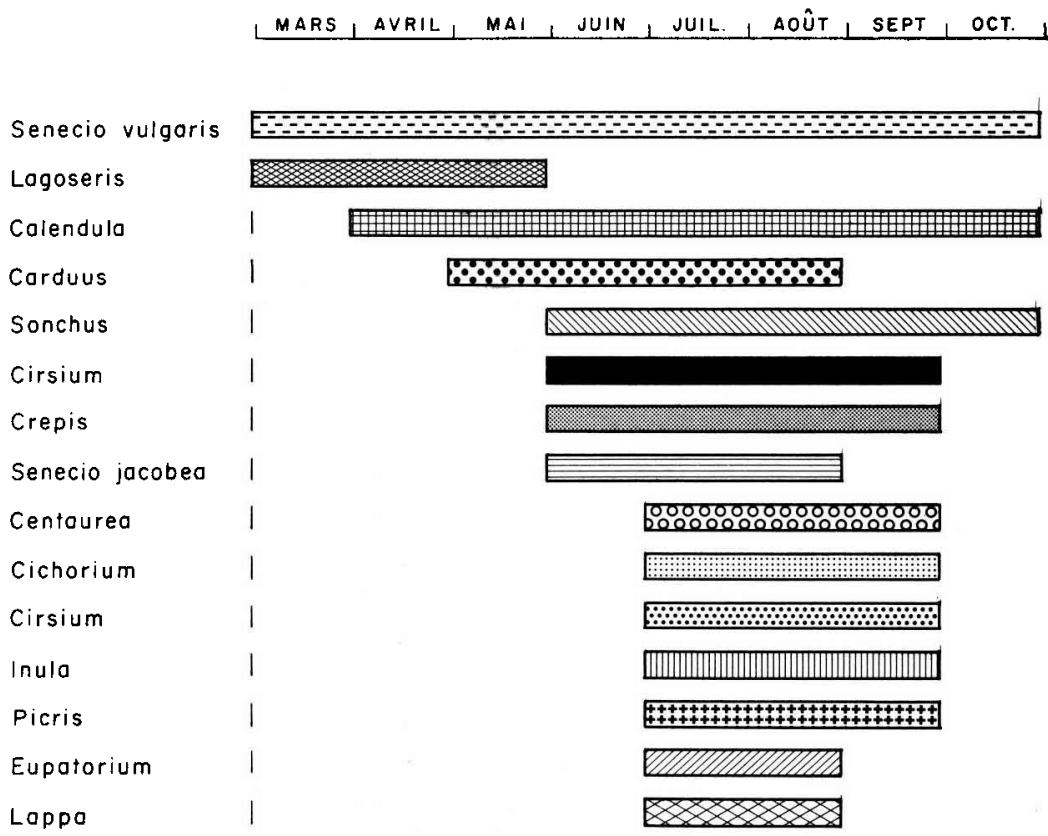

FIG. 9. - Floraisons des principales espèces végétales butinées par Heriades truncorum $L$. dans les huit stations de piégeage.

Авв. 9. - Blütezeit der wichtigsten von Heriades truncorum $L$. besuchten Pflanzenarten an den 8 Versuchsstationen. 
Malgré le grand nombre d'espèces de plantes présentes à chaque station, nous remarquons que les pollens récoltés au cours des années appartiennent uniquement à la famille des Composées. Le caractère oligolectique de l'espèce est ainsi bien confirmé (fig. 10 et 11). Nous trouvons parmi les espèces recherchées trois catégories principales de pollen :

1) Le type de pollen fenestré auquel appartiennent les genres Picris, Crepis, Cichorium et Lagoseris.

2) Le type "Inula" regroupant les genres Senecio, Matricaria, Eupatorium.

3) Les pollens de Lappa (=Arctium), Cirsium, Centaurea, qui ont une morphologie bien distincte des deux grands types précédents.

Les pollens dominants sont toujours Senecio et Inula auxquels s'associe souvent un pollen de type Crepis (planche I).

Parmi les Composées, on constate une préférence bien nette pour celles qui ont des fleurs jaunes, surtout les genres Inula, Crepis, Senecio, Picris. Deux années de suite (1974 et 1975) nous avons proposé dans les cages les espèces suivantes : Senecio jacobaea L., Calendula sp., Cichorium intybus L., Inula dysenterica L., et Picris hieracoïdes L. En 1974, les insectes ont montré une préférence bien marquée pour les plantes à capitules jaunes (Senecio, Picris, Inula). Un faible pourcentage de grains de pollen de Cichorium (fleurs bleues) a été trouvé dans les provisions. Par contre en 1975, leur choix préférentiel s'oriente uniquement vers Inula et Picris, les autres espèces étant délaissées.

D'après nos observations en cage, le nectar est récolté sur les fleurs de Composées. Des plantes telles que Daucus carota L., Hypericum, Agrimonia ont été placées dans les cages, mais nous n'avons jamais observé la récolte du nectar de ces plantes.

- Variations des récoltes en fonction des lieux.

Par l'observation de notre relevé partiel des espèces végétales herbacées, spontanées ou cultivées, récoltées dans un rayon de $300 \mathrm{~m}$ autour des sites de piégeage, nous constatons que :

- Au lieu-dit "Le Chêne " malgré la fréquence relative des espèces, fréquence notée par une, deux ou trois croix, les espèces retrouvées dans l'analyse pollinique sont par ordre décroissant : Senecio jacobaea, Crepis virens, Picris hieracoïdes, Lappa, Lagoseris nemausensis.

- A Jazeneuil l'espèce Inula dysenterica se trouve rarement dans un rayon à 100, $200 \mathrm{~m}$ des nichoirs. Pourtant le pollen de cette espèce est le plus abondant dans les provisions. Ensuite nous avons trouvé par ordre décroissant : Senecio jacobaea, Crepis, Picris. 


\section{2}

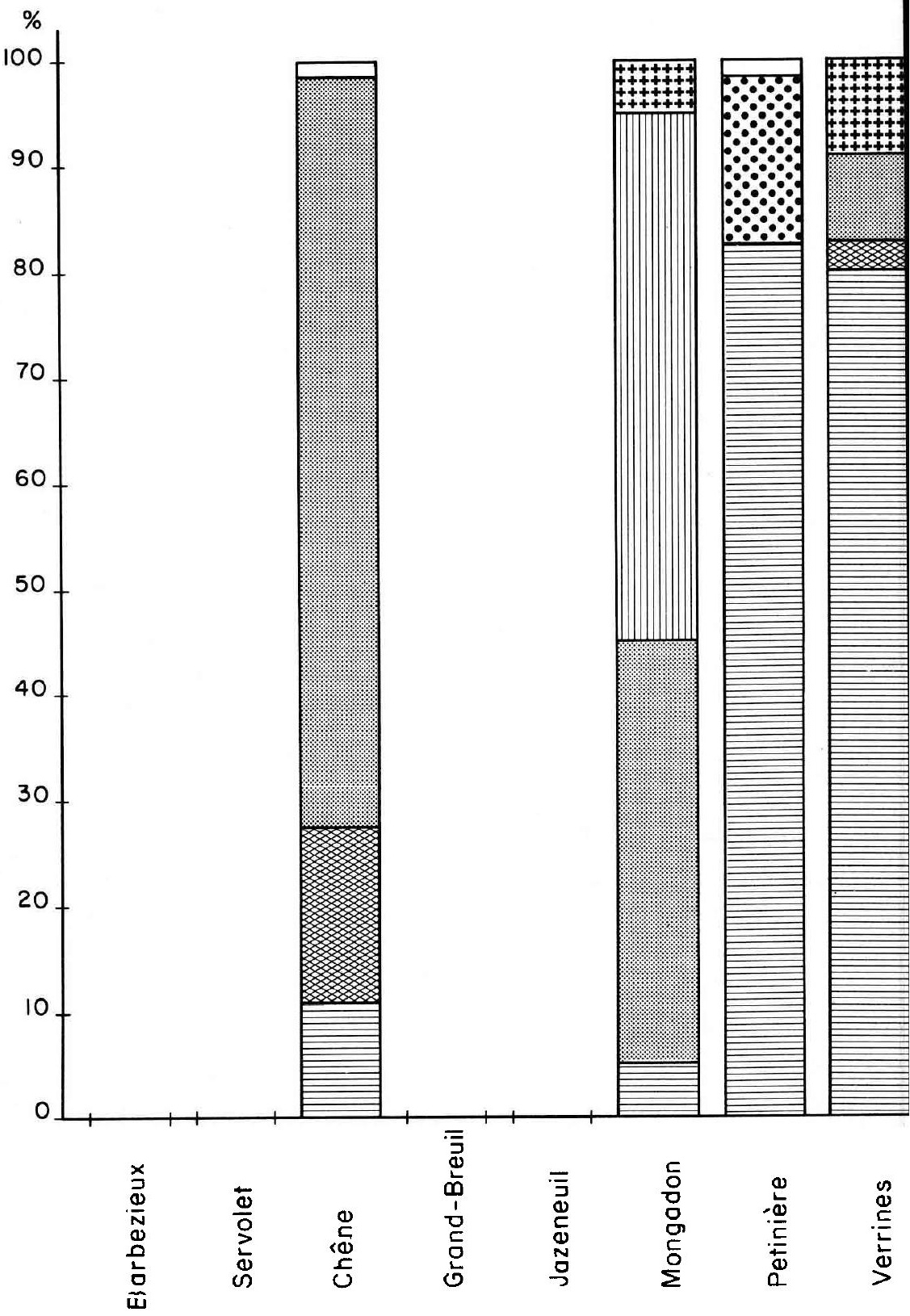

Fig. 10. - Proportion des pollens dans les cellules d'Heriades truncorum $L$. selon les lieux. Même légende que la figure 9.

Année 1972 : L'observation porte sur 22 nids, soit 32 cellules. Année 1973 : L'observation porte sur 30 nids, soit 44 cellules. 
1973
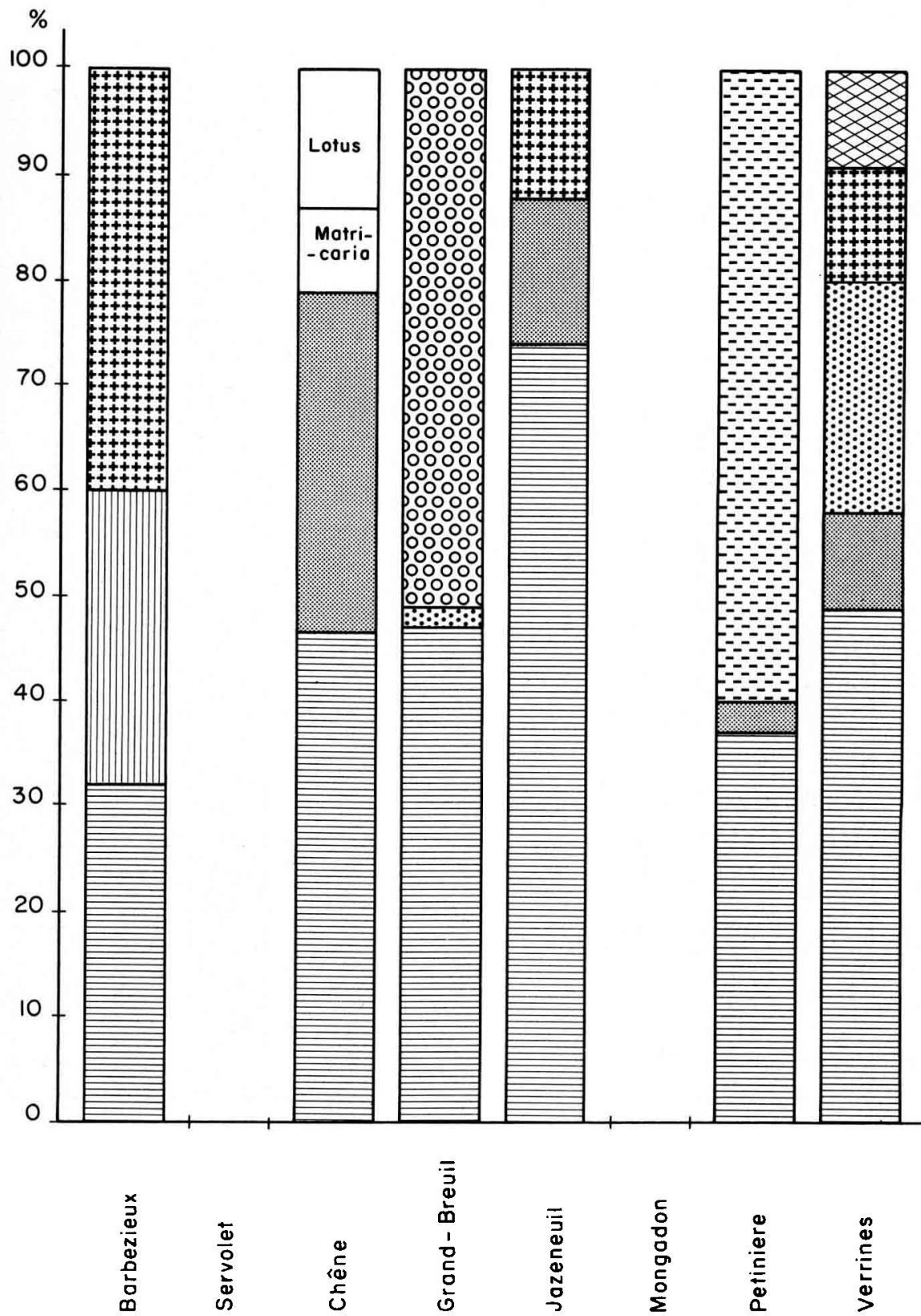

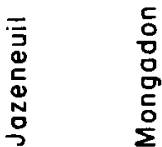
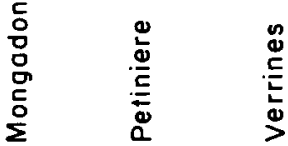

AвB. 10. - Anteil der Pollensorten in den Zellen von Heriades truncorum L. geordnet nach Versuchsorten. Symbole wie in Abb. 9. 1972 : Die Beobachtung umfasst 22 Nester mit 32 Stellen. 1973 : Die Beobachtung umfasst 30 Nester mit 44 Stellen. 


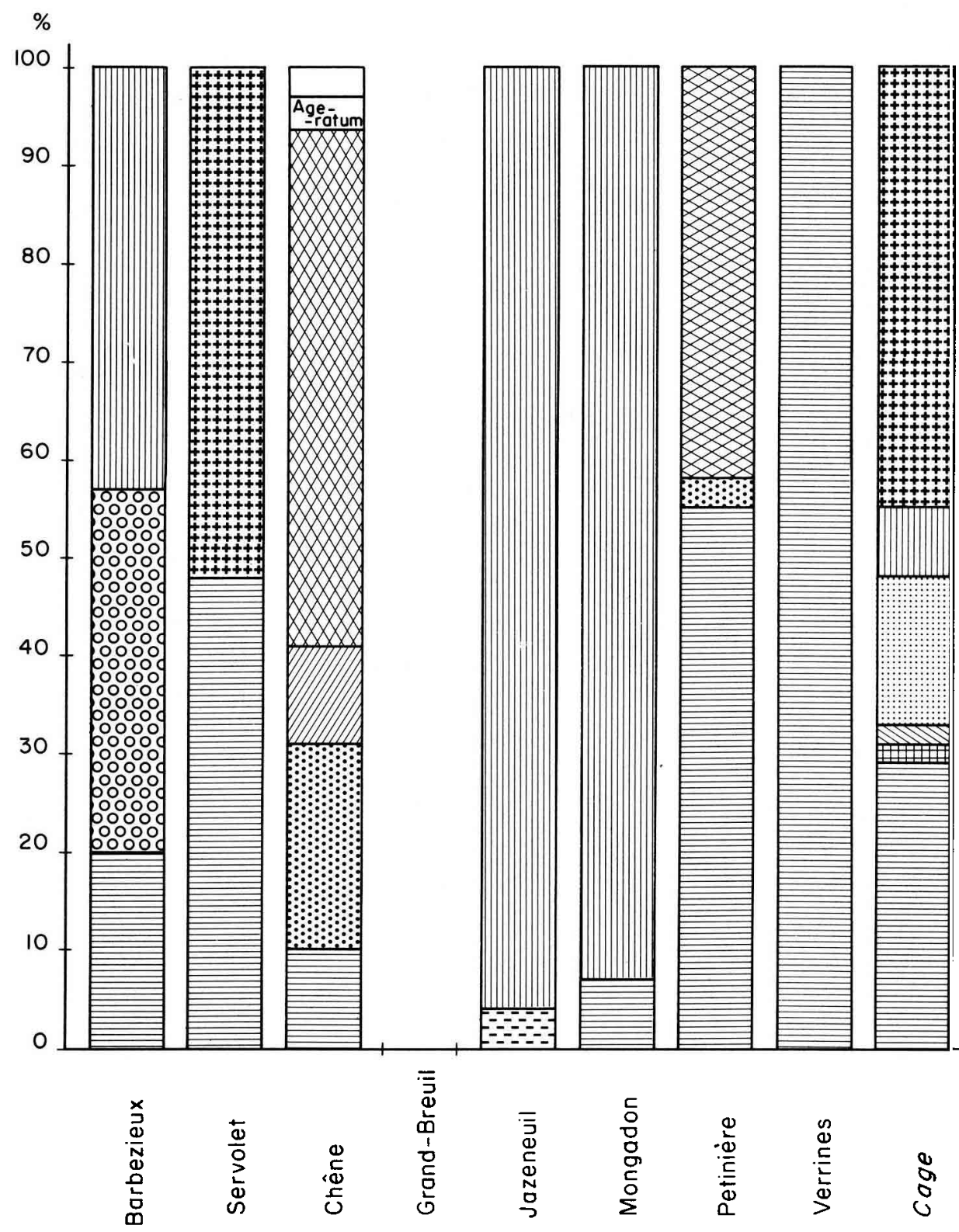

FIG. 11. - Proportion des pollens dans les cellules d'Heriades truncorum L. selon les lieux. Même légende que la figure 9 . Année 1974 : L'observation porte sur 33 nids, soit 83 cellules. Année 1975 : L'observation porte sur 17 nids, soit 49 cellules. 
1975

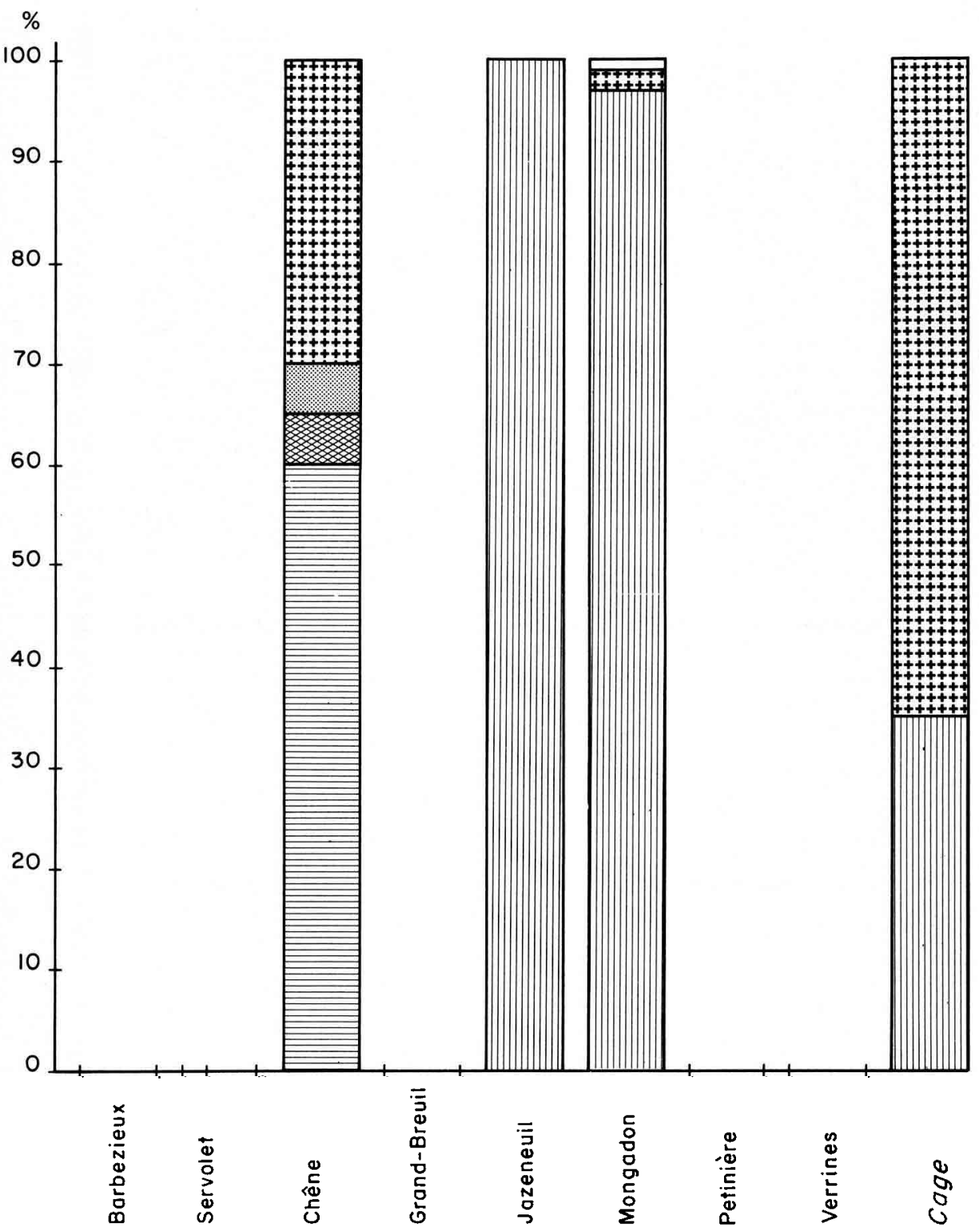

Авв. 11. - Anteil der Pollensorten in den Zellen von Heriades truncorum L. geordnet nach Versuchsorten.

Dieselben Symbole wie in Abb. 9.

1974 : Die Beobachtung umfasst 33 Nester mit 83 Stellen.

1975 : Die Beobachtung umfasst 17 Nester mit 49 Stellen. 

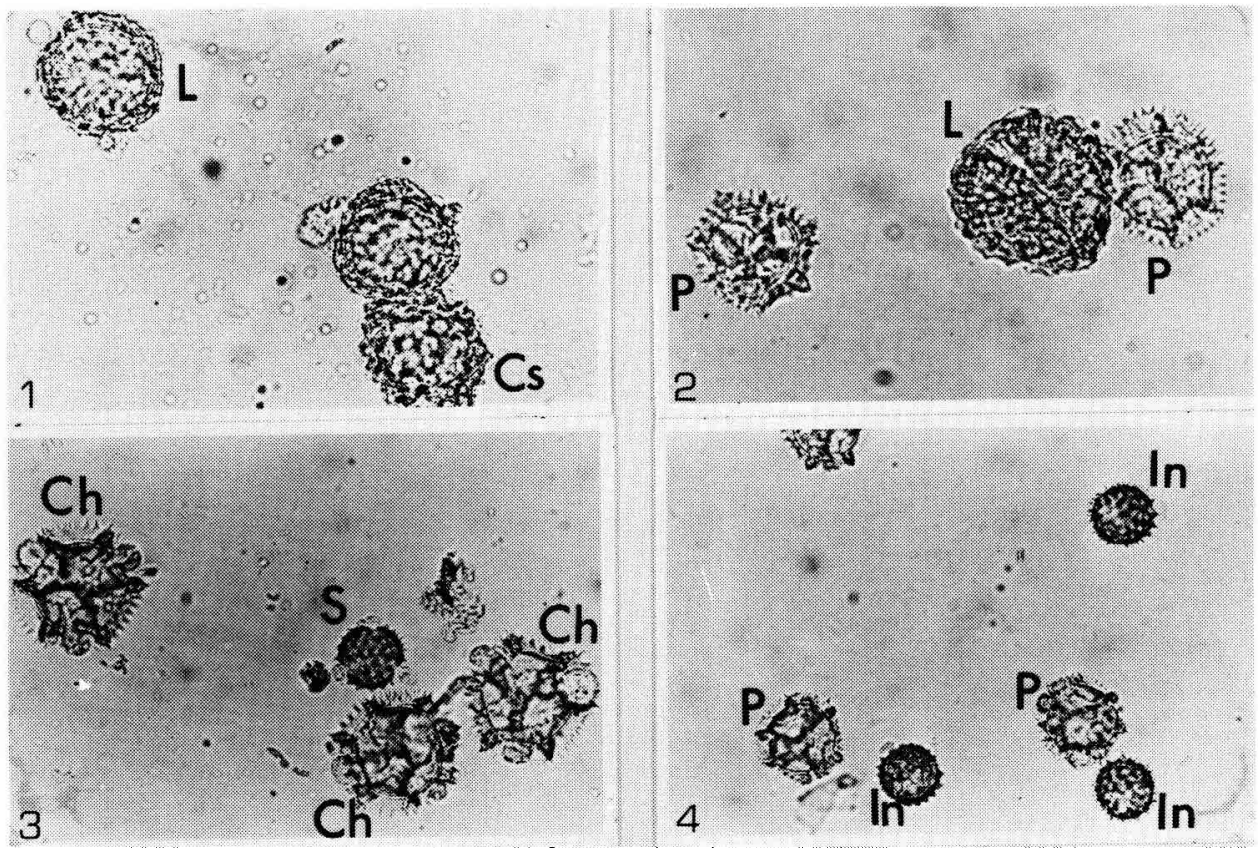

Planche I. - Principaux pollens trouvés dans les provisions polliniques d'Heriades truncorum $L$.
Ch. = Cichorium.
Cs. = Cirsium
In. = Inula.
L. = Lappa.
P. = Picris.
S. = Senecio.

Grossissements : $1=\times 500$.

$2=\times 630$.

$3,4=\times 400$.

TAFEL I. - Wichtigste Pollensorten in den Pollenvorräten von Heriades truncorum $L$.
Ch. = Cichorium.
Cs. = Cirsium.
In. = Inula.
L. = Lappa.
P. = Picris.
S. = Senecio.

$$
\text { Vergrösserungen : } \begin{aligned}
1 & =\times 500 . \\
2 & =\times 630 . \\
3,4 & =\times 400 .
\end{aligned}
$$

- A Mongadon les espèces Eupatorium canabinum, Inula dysenterica, Senecio jacobaea, Lagoseris nemausensis et Taraxacum sont également abondantes dans cette région. Cependant la préférence des insectes est nette : Inula, Crepis, Senecio et Picris.

- A La Pétinière les espèces les plus fréquentes sont Cichorium intybus et Crepis tinctorum. Toutes les autres sont rares. Cependant, les insectes ont plutôt recherché les genres Senecio, Lappa, Carduus, Crepis et Cirsium. 
- Aux Verrines l'espèce Cirsium lanceolatum est la plus fréquente. Toutefois celle qui est la plus recherchée est Senecio jacobaea secondairement Cirsium, Crepis et Lappa.

- A Servolet nos observations se limitent à la seule année de nids construits en 1974. Les espèces les plus abondantes sont Centaurea pratensis, Crepis tinctorum et Picris hieracoïdes. Les espèces moyennement abondantes sont Crepis virens et Senecio jacobaea. Cependant la préférence de pollen est surtout obtenue avec Senecio jacobaea, l'insecte choisit ensuite Picris hieracoïdes.

- Variations des récoltes en fonction des années et des mois.

Le pourcentage des pollens trouvés au cours des années est schématisé sur la figure 12. Nous constatons qu'en 1972 les espèces les plus recherchées sont Crepis virens L. et surtout Senecio jacobaea. En 1973 prédomine le genre Senecio (S. jacobaea et vulgaris). Les genres Crepis, Picris, Inula sont récoltés de façon secondaire. Par contre, au cours des années 1974 et 1975, c'est Inula dysenterica L. qui est l'espèce la plus fréquemment trouvée dans les nids.

Si l'on considère les résultats globalement nous pouvons conclure que : en juillet, le genre le plus récolté est Senecio; Crepis et Centaurea sont moins abondamment récoltés. Au mois d'août, pendant les années 1972 et 1973, les genres préférés sont Senecio et Crepis. Au cours des années 1974 et 1975 le genre le plus butiné est Inula. Toutefois en 1974, l'éventail de genres butinés est assez large. L'insecte recherche également Senecio, Picris, Lappa et dans des proportions moindres Cirsium, Cichorium, Eupatorium.

En septembre, et au cours des quatre années observées l'abondance des pollens par genre est semblable à celle du mois précédent.

- Provisions uniflorales ou multiflorales.

Finalement, il était intéressant de connaître la fréquence des provisions uni ou multiflorales trouvées dans les cellules. Nos observations sont rapportées sur le tableau 4. La majorité $(56,7 \%)$ des provisions contiennent un seul pollen; $23,5 \%$ des provisions contiennent deux sortes de pollens. Il n'y a que $0,5 \%$ de cellules approvisionnées avec 5 pollens différents.

En $1972,40,6 \%$ des provisions sont constituées par plusieurs pollens alors que $37,5 \%$ sont uniflorales. Au cours des années 1973, 1974 et 1975, les provisions uniflorales sont plus nombreuses représentant respectivement $36,3 \%, 59 \%$ et $83,6 \%$ du total des provisions.

\section{CONCLUSION ET DISCUSSION}

La méthode de piégeage permet d'évaluer la variation mensuelle et annuelle des populations, la variation par lieu et la période de vol de l'espèce. La technique du piège- 


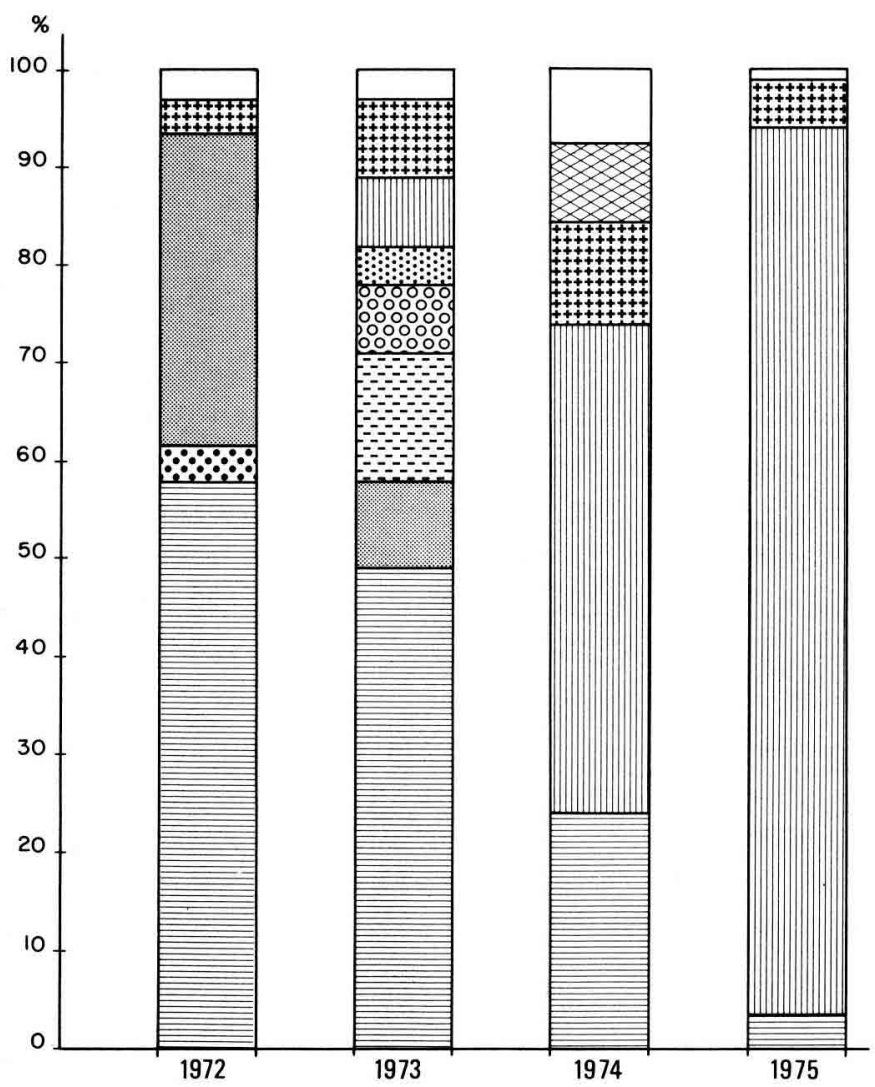

FIG. 12. - Pourcentage des pollens trouvés dans les cellules d'Heriades truncorum $L$. selon les années.

Même légende que la figure 9.

Seuls les effectifs supérieurs à $2 \%$ figurent dans le tableau.

AвB. 12. - Anteil der Pollensorten in den Zellen von Heriades truncorum $L$. geordnet nach Jahren.

Dieselben Symbole wie in Abb. 9.

Nur Mengen über $2 \%$ sind auf der Abbildung dargestellt.

nichoir à tubes démontables permet aussi d'analyser le contenu des charges polliniques et des matériaux de construction.

A l'émergence (dès juin), les mâles apparaissent quelques jours (une à deux semaines) avant les femelles. L'accouplement a lieu dès que ces dernières sortent de leur cocon, la nidification commence 3 à 4 jours après. Les femelles recherchent des cavités tubulaires de 3 à $4 \mathrm{~mm}$ de diamètre orientées de préférence vers le sud et l'est. Si l'on considère huit orientations, on constate que $62,2 \%$ des nids sont construits dans les 
TABL. 4. - Fréquence des cellules contenant des provisions uniflorales ou multiflorales. ТАв. 4. - Häufigkeit der Zellen mit Pollenvorräten von einer einzigen oder von mehreren Pflanzenarten.

\begin{tabular}{|c|c|c|c|c|c|c|}
\hline $\begin{array}{r}\text { Nombre d'espèces florales } \\
\text { par cellule } \\
\text { (pollens) } \\
\text { Année } \\
\text { Jahr }\end{array}$ & 1 & 2 & 3 & 4 & 5 & Total \\
\hline 1972 & 12 & 13 & 4 & 3 & - & 32 \\
\hline 1973 & 16 & 13 & 11 & 3 & 1 & 44 \\
\hline 1974 & 49 & 19 & 12 & 3 & - & 83 \\
\hline 1975 & 41 & 4 & 4 & - & - & 49 \\
\hline Total & 118 & 49 & 31 & 9 & 1 & 208 \\
\hline$\%$ & 56,7 & 23,5 & 15 & 4,3 & 0,5 & \\
\hline
\end{tabular}

nichoirs orientés au sud, à l'est et au sud-est. L'activité commence tard le matin $\left(10 \mathrm{~h} \mathrm{30}\right.$, heure solaire), la température seuil variant de 19 à $24^{\circ} \mathrm{C}$. En moyenne, leur activité journalière dure 6 à 7 heures sous des conditions climatiques favorables.

Les mâles ne récoltent pas de pollen. Les femelles butinent sur les Composées comme nous l'avons signalé précédemment. Leur activité de nidification est donc synchronisée avec la floraison d'un grand nombre de ces plantes. Elles ont une préférence marquée pour celles qui ont des fleurs jaunes. La cadence de butinage est de 8 à $12 \mathrm{ca}$ pitules visités par minute.

La nidification étant plus abondante dans certains lieux par rapport à d'autres, nous avons été conduits à analyser les facteurs d'abondance tels que la végétation et la source des matériaux de construction des nids.

De nos observations (tableau 5) il ressort que les pollens récoltés de préférence sont : Senecio, Inula, Cepis, Picris. Cependant, Senecio est le seul qui soit récolté en abondance en tout lieu. Ces résultats ont été confirmés en serre; lorsque l'on fournit en même temps 3 ou 4 espèces de Composées simultanément, les femelles butinent de préférence Senecio.

D'après les observations de KÄPYLÄ (1978), les principaux pollens récoltés sont du type Achillea (Achillea sp., Matricaria sp., Chrysanthemum sp., Anthemis sp.). De façon secondaire, $H$. truncorum L. récolte des pollens du type Liguliflorae (Taraxacum, Hieracium, Leontodon, Crepis, Sonchus, Lampsana). Toutefois, la plupart des charges contiennent des mélanges des deux types de pollen. Occasionnellement Heriades truncorum $\mathrm{L}$. récolte Cirsium arvense. 


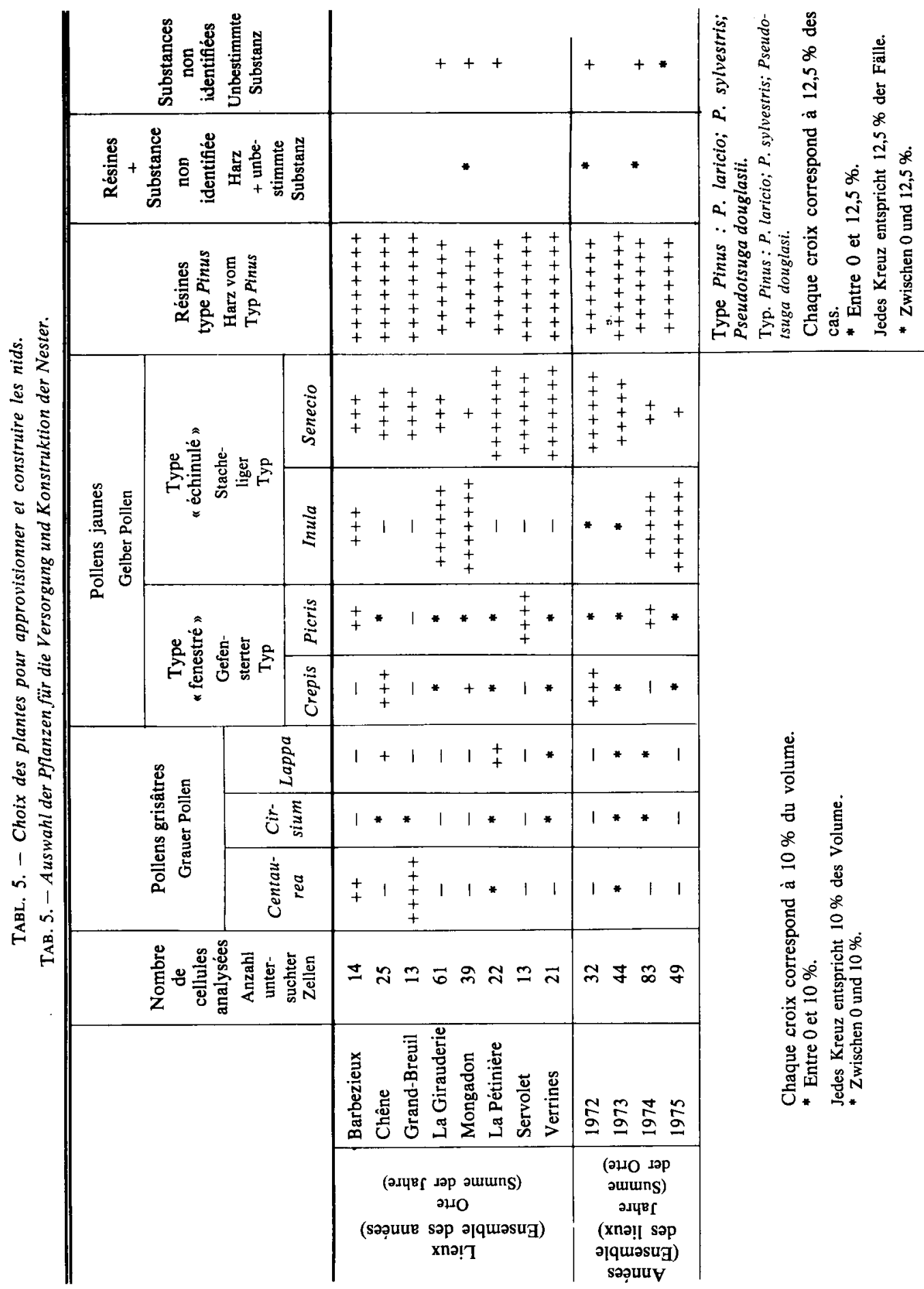


En conclusion de cette étude, on peut considérer que le pollen type recherché par Heriades truncorum $\mathrm{L}$. est un pollen de Composée tubiflore à fleurs jaunes, de taille moyenne à petite ( 20 à $30 \mu$ ), et de préférence échinulé ou plus rarement fenestré.

Étant donné la prédominance nette des pollens répondant à cette description dans les récoltes que nous avons analysées on est en droit de considérer que les pollens appartenant à d'autres genres, Centaurea, Cichorium, Cirsium, Lappa, ne sont que des pollens de substitution dont la récolte peut fort bien être la conséquence de l'absence locale du pollen type, décrit plus haut. Cette hypothèse fait apparaitre Heriades truncorum L. comme particulièrement oligolectique. Soulignons que chez les Megachilidae, l'oligolectie présente divers degrés :

- Le sommet de la spécialisation se rencontre chez Osmia adunca butinant uniquement Viperine.

- Osmia coerulescens est moyennement oligolectique car recherchant surtout les Papilionacées, elle butine secondairement les Labiées.

- Heriades truncorum L. est un cas intermédiaire semblable à Osmia californica butinant également et seulement les Composées.

La confrontation des relevés partiels des espèces avec les résultats de nos analyses polliniques nous amène à penser que des recherches nouvelles devraient être entreprises sur le comportement de butinage de l'insecte, car on comprend mal que le pollen type pratiquement toujours présent dans tous les lieux et tous les ans, ne soit pas préféré dans la totalité des cas, aux pollens de substitution. Une autre hypothèse à envisager serait l'existence de caractères adaptatifs génétiques au sein des populations étudiées. Cette hypothèse permettrait de mieux comprendre les divergences enregistrées entre nos résultats et ceux de KäPYLÄ (1978).

Pour assurer la construction du nid, la présence de Conifères s'avère primordiale. Une publication traitant de ce sujet (MACIEL CoRreIA, 1977) ainsi que des résultats postérieurs indiquent la nécessité pour les femelles de trouver dans les environs du site de nidification des Conifères fournissant la résine indispensable à l'édification des cloisons et bouchons des nids. En effet, $94 \%$ des 311 analyses des matériaux de construction montrent que la résine est empruntée aux espèces : Pinus laricio Poir., Pinus silvestris L. Pseudotsuga menziesii (Mich) Franco ( $=P$. douglassii Carr.). Cette forte proportion est à peu près constante d'un lieu à l'autre (tableau 5).

Il faut savoir que la résine de Cyprès n'est jamais employée; par contre, en serre, les femelles ont récolté de la résine d'Abies alba Mill. $=A$. pectinata $(\mathrm{Lam}$.) DC. et Picea abies $(\mathrm{L}$.) Kars $=P$. excelsa $(\mathrm{Lam}$.) Link (substances non analysées en chromatographie) mais avec moins de fréquence que les espèces précédentes, elles ont également récolté du mastic à joint. Environ $6 \%$ des constructions sont dues à des substances non identifiées, dans trois lieux sur huit seulement et trois années sur quatre.

Selon MatTHews (1965) et chez Heriades carinata Cress., la résine employée dans les cloisons et les bouchons est pure. Chez Heriades variolosus (Cress.), (Fischer, 
1955) la femelle y incorpore des particules de sable et de terre. MiChener (1968), après la description d'un nid d'Heriades (Heriades) spiniscutis (Cameron), signale sa principale caractéristique qui est parfois l'absence de cloisons séparant les cellules. Quand ces dernières existent, elles sont formées 'de résine d'origine inconnue. Toutefois, ces Heriades ont été observés sur des Euphorbia; elles y récoltaient du latex coagulé, pour la construction du nid.

Aussi, il serait intéressant de préciser l'influence et la hiérarchie de certains facteurs du milieu : l'abondance des plantes nourricières, leur valeur nutritive (qualitativement et quantitativement), l'accessibilité du pollen et du nectar, et la présence de plantes fournissant le matériau de construction.

Dans certaines localités étudiées et certaines années, nous avons constaté que les vols d'Heriades truncorum $\mathrm{L}$. étaient très réduits alors que les conditions de température et d'alimentation étaient idéales. La flore et les conditions climatiques estivales ne sont pas les seuls facteurs responsables de l'épanouissement des populations de cette espèce.

L'étude de certains facteurs climatiques tels que la température (maximale et minimale) et la pluviométrie, ne nous permet pas de mettre en évidence leur influence directe sur la nidification. Toutefois, cela nous amène à conclure que, dans des études futures, l'influence d'autres facteurs de climat (durée de l'exposition des nichoirs au soleil, écart des températures diurnes et nocturnes) devra être prise en considération. Aussi les taux de mortalité liés au parasitisme, à la prédation et aux conditions d'hibernation doivent être pris en considération pour expliquer ces variations.

Si fragmentaires que soient les relations que nous avons pu établir, tout concorde pour faire admettre que la plupart des femelles choisissent des nichoirs-pièges à des emplacements définis par des facteurs essentiellement trophiques (présence de Composées) - la part qui revient aux facteurs microclimatiques restant à déterminer par d'autres méthodes analytiques.

\section{REMERCIEMENTS}

Nous remercions très sincèrement $M$. TASEI qui a bien voulu se charger de la détermination des pol lens et de donner son appui tout au long de ce travail,

M. LouveauX pour ses conseils dans la rédaction du manuscrit,

M. GUY et M. GENIER (I.N.R.A., Lusignan) pour leurs conseils lors de l'analyse statistique de certains résultats,

M. BARon (Faculté des Sciences de Poitiers) et M. Lemaire (I.N.R.A., Lusignan) pour l'identification de certaines espèces végétales,

M. CARre pour l'exécution des photos,

M. BRANCo pour son appui lors de la mise au point de ce travail.

Reçu pour publication en juillet 1980.

Eingegangen in Juli 1980. 


\section{ZUSAMMENFASSUNG}

In einem früheren Beitrag (MACIEL CoRreiA, Apidologie, 1980, 4, 309-339) wurde der biologische Zyklus der Art Heriades truncorum L. beschrieben. Es wurde seine monovoltine Generationsfolge (eine Generation im Jahr) nachgewiesen sowie die verschiedenen Larvenstadien unterschieden.

Jetzt wird der entscheidende Einfluss des äusseren Milieus auf die Wahl des Nistplatzes und die Auswahl der Röhrchen in Abhängigkeit von ihrem Durchmesser untersucht.

An sieben Plätzen, die verschiedene Typen von Niststätten darstellten und mit Hilfe künstlicher Nistgelegenheiten wurde für Heriades truncorum L. die Variation über die Monate und Jahre und die Variation nach Nistplatz ausgewertet.

Es wurden die Kriterien bei der Wahl des Nistplatzes analysiert. Die Weibchen schienen sehr wählerisch bei der Auswahl der Nester in Bezug auf ihre Richtung und bei der Auswahl der Niströhrchen in Bezug auf ihren Durchmesser. Sie wählten bevorzugt Nistplätze mit Orientierung nach Süden und Osten und nisteten bevorzugt in Röhrchen mit $3 \mathrm{~mm}$ Durchmesser.

Da die Nester an manchen Orten zahlreicher waren als an anderen, hat der Autor zwei die Häufigkeit beeinflussende Faktoren untersucht : Den Ursprung des Nestbaumaterials und die Herkunft von Nektar und Pollen. In einer ersten Phase erfolgte eine Inventarisierung der Flora in einem Umkreis von 200-300 m um die Nistplätze; dies ergab Aufschluss über die charakteristischen Arten in jedem Milieu. In einer zweiten Phase wurden Pollenvorräte aus Zellen von Nestern in den verschiedenen Nistplätzen untersucht, um die Pflanzen ausfindig zu machen, an denen die Weibchen Pollen sammeln.

Die Analysen umfassten 208 Zellen und sie erlauben folgende Schlüsse : Gelber stachliger Pollen (Inula, Senecio) gefolgt von Pollen mitgefensterter Oberfläche (Picris, Crepis) wurde grauem Pollen (Centaurea, Cirsium, Lappa) vorgezogen. Nur an einem einzigen Ort (Grand-Breuil) wurde grauer Pollen (Grand-Breuil) wurde grauer Pollen stärker gesammelt als gelber Pollen.

Die Methode der mikroskopischen Pollenanalyse hat sich demnach als urumgänglich für den Nachweis erwiesen, dass Heriades truncorum L. beim Sammeln ein typischer Spezialist (oligolektisch) ist - diese Biene interessiert sich nur für Pflanzen aus der Familie der Kompositen - ein seltener Fall bei den Apoidea.

Uniflorale Vorräte sind am häufigsten : $56,7 \%$. Vorräte mit zwei Pollensorten wurden in $23,5 \%$ der Zellen angetroffen, während Vorräte mit fünf verschiedenen Pollensorten nur $0,5 \%$ des Untersuchungsmaterials ausmachten.

\section{BIBLIOGRAPHIE}

Arbonnier P., 1967 - L'analyse de l'information. Aperçu théorique et application à la loi multinomiale. Ann. Sc. Forest. 23 (4), 951-1016.

ERdtMan G., 1943 - An introduction to pollen analysis. Chronica Botanica, Waltham, Mass., $239 \mathrm{p}$.

JAEGER P., 1976 - Les rapports mutuels entre fleurs et insectes, in Traité de Zoologie de Grassé, 8 (4), 677-798.

JohANSEN C.A., Eves J.D., 1973 - Effects of chilling, humidity and seasonal conditions on emergence of the alfalfa leafcutting bee. Environ. Entomol., 2, 23-26.

JOURDHEUIL P., 1961 - Influence de quelques facteurs écologiques sur les fluctuations de population d'une biocénose parasitaire. Thèse Fac. Sc. Univ., Paris.

KÄPYLÄ M., 1978 - Bionomics of five wood-nesting solitary species of bees (Hymenoptera, Megachilidae), with emphasis on flower relationships. Biol. Res. Rep. from Univ. of Jyväskylä., 1-89.

Leсомте J., 1962 - Techniques d'étude des populations d'insectes pollinisateurs. Ann. Abeille, 5 (3), 201213.

LeviN M.D., 1957 - Artificial nesting burrows for Osmia lignaria Say. J. Econ. Ent., 50 (4), 506-507. 
LeviN M. D. and HAYDAK M. H., 1957 - The comparative value of different pollens in the nutrition of Osmia lignaria Say. Bee World, 38 (9), 221-226.

LINSLEY E.G., 1958 - The ecology of solitary bees. Hilgardia, 27, 543-599.

LouveauX J., 1958-1959 - Recherches sur la récolte du pollen par les abeilles (Apis mellifica L.). Ann. A beille, 1958, 3, 113-188; 4, 197-221 et 1959, 1, 13-111.

LouveAuX J., 1976 - Biologie florale, pollinisation et évolution. Bull.tech. Apic. OPIDA, 3 (2), 19-83.

Louveaux J., Vergeron Ph., 1964 - Étude du spectre pollinique de quelques miels. Ann. Abeille, 7, 329. 347.

Louveaux J., Maurizio A., VoRwohl G., 1970 - Internationale Kommission für Bienenbotanik der I.U.B.S. Methodik der Melissopalynologie. Apidologie, 1, 193-209.

MAciel de A. Correia M., 1976 - Notes sur la biologie d'Heriades truncorum L. (Hymenoptera, Megachilidae). Apidologie, 7 (2), 169-187.

Maciel de A. Correia M., 1977 - Sur l'origine des résines employées par Heriades truncorum L. (Hymenoptera, Megachilidae) pour la construction de ses nids. Apidologie, 8 (2), 101-109.

MatThews R. W., 1965 - The biology of Heriades carinata Cress. (Hymenoptera, Megachilidae). Thesis Ph. D. Contrib. Amer. Ent. Inst., 1 (3), 1-33.

MiChENER C.D., 1968 - Heriades spiniscutis, a bee that facultatively omits partitions between rearing cells (Hymenoptera, Apoïdea), Journ. Kans. Ent. Soc., 41, 484-493.

Robertson C., 1914 - Origin of oligolectic bees and favorite flowers. Psyche, 36, 112-118.

Robertson C., 1925 - Heterotropic bees. Ecology, 6, 412-436.

TASEI J.-N., 1973 - Le comportement de nidification chez Osmia (Osmia) cornuta Latr. et Osmia (Osmia) rufa L. (Hymenoptera, Megachilidae). Apidologie, 4 (3), 195-225.

TASEI J.-N., 1976 - Récolte des pollens et approvisionnement du nid chez Osmia coerulescens L. (Hyme. noptera, Megachilidae). Apidologie, 7, 277-300. 